\title{
AN ESTIMATE FOR THE MEAN CURVATURE OF COMPLETE SUBMANIFOLDS IN A TUBE
}

\author{
By Yoshinisa Kitagawa
}

\section{Introduction.}

Let $f: M \rightarrow E^{n}$ be an isometric immersion of a compact Riemannian manifold $M$ into the Euclidean space $E^{n}$. If $f(M)$ is contained in a ball of radius $\lambda$, then the mean curvature vector field $H$ of the immersion $f$ satisfies the following inequality :

$$
\sup |H| \geqq 1 / \lambda .
$$

Recently, generalizing the above inequality, Jorge and Xavier [4], and Jorge and Koutroufiotis [2] proved the following theorem.

THEOREM A. Let $M$ be a complete Riemannian manifold whose scalar curvature is bounded below and let $B_{\lambda}$ be a closed normal ball of radius $\lambda$ in a Riemannian manifold $N$. Set $b$ for the supremum of the sectional curvature of $N$ in $B_{\lambda}$. Let $f: M \rightarrow B_{\lambda} \subset N$ be an isometruc immersion. Then the mean curvature vector field $H$ of the immersion $f$ satisfies the following:

(1) $\sup |H| \geqq \sqrt{b} / \tan (\lambda \sqrt{b})$, if $b>0$ and $\lambda<\pi / 2 \sqrt{b}$,

(2) $\sup |H| \geqq 1 / \lambda$, if $b=0$,

(3) $\sup |H| \geqq \sqrt{-b} / \tanh (\lambda \sqrt{-\bar{b}})$, if $b<0$.

In this paper we show a natural extensior of the inequalities in Theorem $\mathrm{A}$ considering a tube instead of a ball.

\section{Statement of results.}

Let $f: M \rightarrow N$ be an isometric immersion of an $m$-dimensional complete Riemannian manifold $M$ whose scalar curvature is bounded below into an $n$-dimensional Riemannian manifold $N$ whose sectional curvature $K_{N}$ satisfies $-\infty<\inf K_{N}$ and $K_{N} \leqq b$. For $n>p \geqq 1$, let $P$ be a $p$-dimensional embedded submanifold in $N$ and let $T P^{\perp}$ be the normal bundle of $P$. We denote $\tau(P, \lambda)$ the tube of radius $\lambda$ about $P$ in $N$ (i.e. $\left\{\xi \in T P^{\perp}:|\xi| \leqq \lambda\right\}$ is mapped diffeomorphically onto $\tau(P, \lambda)$ through the exponential map). We define $\mu$ by

Recenved April 18, 1983 


$$
\mu=\sup \left\{\mu(\xi): \xi \in T P^{\perp},|\xi|=1\right\},
$$

where $\mu(\xi)$ denotes the maximum eigenvalue of the shape operator $A_{\xi}$. Then our main result is the following.

THEOREM B. In the notations above suppose that $f(M)$ is contained in a tube about $P$. Let $\lambda$ be the infimum of the radius of tubes about $P$ which contain $f(M)$. If $p \leqq m$, and $0<\lambda$, then the mean curvature vector field $H$ of the immersion $f$ satisfies the following.

(1) $\sup |H| \geqq-\frac{p}{m}\left(\frac{\mu \sqrt{b}+b \cdot \tan (\lambda \sqrt{b})}{\sqrt{b}-\mu \cdot \tan (\lambda \sqrt{b})}\right)+\frac{m-p}{m}\left(\frac{\sqrt{b}}{\tan (\lambda \sqrt{b})}\right)$,

if $b>0, \lambda<\pi / 2 \sqrt{b}$ and $\mu<\sqrt{b} / \tan (\lambda \sqrt{b})$,

(2) $\sup |H| \geqq-\frac{p}{m}\left(\frac{\mu}{1-\mu \lambda}\right)+\frac{m-p}{m}\left(\frac{1}{\lambda}\right)$, if $b=0$ and $\mu<1 / \lambda$,

(3) $\sup |H| \geqq-\frac{p}{m}\left(\frac{\mu \sqrt{-b}+b \cdot \tanh (\lambda \sqrt{-b})}{\sqrt{-b-\mu \cdot \tanh (\lambda \sqrt{-b})}}\right)+\frac{m-p}{m}\left(\frac{\sqrt{-b}}{\tanh (\lambda \sqrt{-\bar{b}})}\right)$, if $b<0$ and $\mu<\sqrt{-\bar{b}} / \tanh (\lambda \sqrt{-b})$.

Applying Theorem B to minimal immersions, we have immediately:

Theorem C. Let $f: M \rightarrow N$ be an isometric immersion of an m-dimensional complete Riemannian manifold $M$ whose scalar curvature is bounded below into an n-dimensional Riemannian manifold $N$ whose sectional curvature $K_{N}$ satisfies $-\infty<\inf K_{N}$ and $K_{N} \leqq b$. For $n>p \geqq 1$, let $P$ be a $p$-dimensional embedded submanifold in $N$ and let $\tau(P, \lambda)$ be the tube of radius $\lambda$ about $P$. Suppose that $f$ is minimal and $P$ is totally geodesic. Then the following holds:

(1) $f(M) \subset \tau(P, \lambda)$, if $b>0, \lambda<\pi / 2 \sqrt{b}$ and $p\left\{1+\tan ^{2}(\lambda \sqrt{b})\right\}<m$,

(2) $f(M) \oplus \tau(P, \lambda)$, if $b=0$ and $p<m$,

(3) $f(M) \subset P$, if $b<0, p \leqq m$ and $f(M) \subset \tau(P, \lambda)$.

Remark. Let $P$ be a linear subspace of $E^{3}$. It is interesting to study complete minimal surfaces in a tube $\tau(P, \lambda)$. For $\operatorname{dim} P \leqq 1$, Theorems $\mathrm{A}$ and $\mathrm{C}$ imply that $\tau(P, \lambda)$ contains no complete minimal surface whose Gaussian curvature is bounded. For $\operatorname{dim} P=2$, Jorge and Xavier [3] proved that there exists a complete non-flat minimal surface in $\tau(P, \lambda)$.

\section{Preliminaries.}

For $n>p \geqq 1$, let $N$ be an $n$-dimensional Riemannian manifold and let $P$ be a $p$-dimensional embedded submanifold in $N$. The Riemannian metric, Riemannian connection and curvature tensor of $N$ are denoted by $\langle\rangle,, \nabla$ and $R$ respectively. Let $\sigma:[0, \lambda] \rightarrow N$ be a geodesic parametrized by arclength such that $\sigma(0) \in P$ and $\dot{\sigma}(0) \in T_{\sigma(0)} P^{\perp}$, where $T_{\sigma(0)} P^{\perp}$ denotes the normal space to $P$ at $\sigma(0)$. 
Let $L(P, \sigma)$ denote the vector space of all piecewise smooth vector fields along $\sigma$ whose initial value is tangent to $P$. The index form for the pair $(P, \sigma)$ is a symmetric bilinear form $I: L(P, \sigma) \times L(P, \sigma) \rightarrow \boldsymbol{R}$ defined by

$$
I(V, W)=-\left\langle A_{\dot{\sigma}(0)} V(0), W(0)\right\rangle+\int_{0}^{\lambda}\left\{\left\langle\nabla_{\dot{\sigma}} V, \nabla_{\dot{\sigma}} W\right\rangle+\langle R(\dot{\sigma}, V) \dot{\sigma}, W\rangle\right\} d t,
$$

where $A_{\dot{\sigma}(0)}$ denotes the shape operator for $\dot{\sigma}(0)$. A Jacobi field $J \in L(P, \sigma)$ is called a $P$-Jacobi field if it satisfies the following condition:

$$
A_{\dot{\sigma}(0)} J(0)+\left(\nabla_{\dot{\sigma}} J\right)(0) \in T_{\sigma(0)} P^{\perp} .
$$

For $0<t_{0} \leqq \lambda, \sigma\left(t_{0}\right)$ is called a focal point of the pair $(P, \sigma)$ if there exists a nonzero $P$-Jacobi field $J$ along $\sigma$ such that $J\left(t_{0}\right)=0$.

LEMma 3.1 ([1, p. 228]). Suppose that there is no focal point of the pair $(P, \sigma)$. Then for each $V \in L(P, \sigma)$ there exists a unique $P$-Jacobi field $J$ along $\sigma$ such that $J(\lambda)=V(\lambda)$. Furthermore $I(J, J) \leqq I(V, V)$ and equality holds only uf $J=V$.

For $(b, \mu, t) \in R^{3}$, we define $g_{i}(b, \mu, t)$ as follows :

$$
\begin{aligned}
& g_{0}(b, \mu, t)=t, \\
& g_{1}(b, \mu, t)= \begin{cases}\cos (t \sqrt{b})-\mu \cdot \sin (t \sqrt{b}) / \sqrt{b} & \text { if } b>0, \\
1-\mu t & \text { if } b=0, \\
\cosh (t \sqrt{-b})-\mu \cdot \sinh (t \sqrt{-b}) / \sqrt{-b} & \text { if } b<0,\end{cases} \\
& g_{2}(b, \mu, t)= \begin{cases}\sin (t \sqrt{b}) / \sqrt{b} & \text { if } b>0, \\
t & \text { if } b=0, \\
\sinh (t \sqrt{-b}) / \sqrt{-b} & \text { if } b<0 .\end{cases}
\end{aligned}
$$

Let $\left\{E_{0}, E_{1}, \cdots, E_{n-1}\right\}$ be a parallel orthonormal frame field along $\sigma$ such that $E_{0}=\dot{\sigma}$ and $E_{j}(0)$ is tangent to $P$ for $1 \leqq j \leqq p$. Then we have the following.

LEMma 3.2. Let $J$ be a P-Jacobi field along $\sigma$ and let $f_{\jmath}=\left\langle J, E_{\jmath}\right\rangle$. Suppose that $N$ has constant sectional curvature $b$ and the shape operator $A_{\dot{\sigma}(0)}$ has $a$ unique eigenvalue $\mu$. Then $f$, satisfies the following.

$$
f_{j}(t)= \begin{cases}f_{0}^{\prime}(0) g_{0}(b, \mu, t) & \text { if } j=0, \\ f_{j}(0) g_{1}(b, \mu, t) & \text { if } 1 \leqq j \leqq p \\ f_{j}^{\prime}(0) g_{2}(b, \mu, t) & \text { if } \quad p+1 \leqq j \leqq n-1 .\end{cases}
$$

LEMMA 3.3. Suppose that $N$ has constant sectional curvature $b$ and the shape 
operator $A_{\dot{\sigma}(0)}$ has a unique eigenvalue $\mu$. Then there is no focal point of the pair $(P, \sigma)$ if one of the following holds:

(3.1) $b>0, \lambda<\pi / 2 \sqrt{b}$ and $\mu<\sqrt{b} / \tan (\lambda \sqrt{b})$,

(3.2) $b=0$ and $\mu<1 / \lambda$,

(3.3) $b<0$ and $\mu<\sqrt{-b} / \tanh (\lambda \sqrt{-b})$.

Remark. Lemma 3.2 implies Lemma 3.3. In Lemma 3.3, if $\mu \geqq 0$, then the nonexistence of focal points of the pair $(P, \sigma)$ implies one of $(3.1)-(3.3)$.

For $(b, \mu, \lambda)$ which satisfies one of $(3.1)-(3.3)$, we define $h_{i}(b, \mu, \lambda)$ as follows:

$$
\begin{aligned}
& h_{0}(b, \mu, \lambda)=1 / \lambda, \\
& h_{1}(b, \mu, \lambda)= \begin{cases}-\frac{\mu \sqrt{b}+b \cdot \tan (\lambda \sqrt{b})}{\sqrt{b}-\mu \cdot \tan (\lambda \sqrt{b})} & \text { if } \text { (3.1) holds, } \\
-\frac{\mu}{1-\mu \lambda} & \text { if } \text { (3.2) holds, } \\
-\frac{\mu \sqrt{-b}+b \cdot \tanh (\lambda \sqrt{-b})}{\sqrt{-b}-\mu \cdot \tanh (\lambda \sqrt{-b})} & \text { if } \text { (3.3) holds, }\end{cases} \\
& h_{2}(b, \mu, \lambda)= \begin{cases}\sqrt{b} / \tan (\lambda \sqrt{b}) & \text { if } \text { (3.1) holds, } \\
1 / \lambda & \text { if } \text { (3.2) holds, } \\
\sqrt{-b} / \tanh (\lambda \sqrt{-b}) & \text { if (3.3) holds. }\end{cases}
\end{aligned}
$$

For the pair $(P, \sigma)$, let $V^{\iota}(P, \sigma)$ be the subspace of $T_{\sigma(\lambda)} N$ defined by

$$
\begin{aligned}
& V^{0}(P, \sigma)=\operatorname{span}\left\{E_{0}(\lambda)\right\}, \\
& V^{\mathrm{J}}(P, \sigma)=\operatorname{span}\left\{E_{1}(\lambda), \cdots, E_{p}(\lambda)\right\}, \\
& V^{2}(P, \sigma)=\operatorname{span}\left\{E_{p+1}(\lambda), \cdots, E_{n-1}(\lambda)\right\} .
\end{aligned}
$$

LEMMA 3.4. Under the same assumptions as in Lemma 3.3, suppose that one of (3.1)-(3.3) holds. Let $J$ be a P-Jacobr field along $\sigma$. Then

$$
I(J, J)=\sum_{\imath=0}^{2} h_{\imath}(b, \ell, \lambda) \mid V^{\imath}(P, \sigma) \text {-component of }\left.J(\lambda)\right|^{2} .
$$

Proof. Let $f_{\jmath}=\left\langle J, E_{\jmath}\right\rangle$. Then $I(J, J)=\left\langle\left(\nabla_{\dot{\sigma}} J\right)(\lambda), J(\lambda)\right\rangle=\sum_{j=0}^{n-1} f_{j}^{\prime}(\lambda) f_{\jmath}(\lambda)$. By Lemma 3.2 we have

$$
f_{j}^{\prime}(\lambda)=\left\{\begin{array}{lll}
f_{0}(\lambda) h_{0}(b, \mu, \lambda) & \text { if } \quad \jmath=0, \\
f_{\jmath}(\lambda) h_{1}(b, \mu, \lambda) & \text { if } 1 \leqq \jmath \leqq p \\
f_{j}(\lambda) h_{2}(b, \mu, \lambda) & \text { if } \quad p+1 \leqq j \leqq n-1 .
\end{array}\right.
$$


Hence $I(J, J)=h_{0}(b, \mu, \lambda) f_{0}^{2}(\lambda)+h_{1}(b, \mu, \lambda) \sum_{j=1}^{p} f_{\jmath}^{2}(\lambda)+h_{2}(b, \mu, \lambda) \sum_{p<j} f_{\jmath}^{2}(\lambda)$. G.e.d.

Lemma 3.5. Suppose that the sectional curvature $K_{N}$ of $N$ satisfies $K_{N} \leqq b$ and the maximum eigenvalue of $A_{\dot{\boldsymbol{\sigma}}(0)}$ is not larger than $\mu$. If one of (3.1)-(3.3) holds for $(b, \mu, \lambda)$, then each $V \in L(P, \sigma)$ satisfies the following.

$$
I(V, V) \geqq \sum_{\imath=0}^{2} h_{i}(b, \mu, \lambda) \mid V^{\imath}(P, \sigma) \text {-component of }\left.V(\lambda)\right|^{2} .
$$

Proof. Let $N(b)$ denote the $n$-dimensional complete simply connected Riemannian manifold of constant sectional curvature $b$ and let $\tau:[0, \lambda] \rightarrow N(b)$ be a geodesic parametrized by arclength. We construct a $p$-dimensional embedded submanifold $\widetilde{P}$ in $N(b)$ such that $\tau(0) \in \widetilde{P}, \dot{\tau}(0) \in T_{\tau(0)} \widetilde{P}^{\perp}$ and the shape operator $A_{\dot{\tau}(0)}$ has a unique eigenvalue $\mu$. Let $\left\{\tilde{E}_{0}, \cdots, \tilde{E}_{n-1}\right\}$ be a parallel orthonormal frame field along $\tau$ such that $\widetilde{E}_{0}=\dot{\tau}$ and $\tilde{E}_{\jmath}(0)$ is tangent to $\widetilde{P}$ for $1 \leqq \jmath \leqq p$. We define $\tilde{V}$ in $L(\tilde{P}, \tau)$ by $\tilde{V}=\sum_{\jmath=0}^{n-1}\left\langle V, E_{\jmath}\right\rangle \tilde{E}_{\jmath}$. Since $K_{N} \leqq b$ and the maximum eigenvalue of $A_{\dot{\sigma}(0)}$ is not larger than $\mu$, we have $I(V, V) \geqq \tilde{I}(\tilde{V}, \tilde{V})$, where $\tilde{I}$ denotes the index form for the pair $(\tilde{P}, \tau)$. By Lemmas 3.1, 3.3 and 3.4 we have

$$
\tilde{I}(\tilde{V}, \tilde{V}) \geqq \sum_{\imath=0}^{2} h_{\imath}(b, \mu, \lambda) \mid V^{\imath}(\tilde{P}, \tau) \text {-component of }\left.\tilde{V}(\lambda)\right|^{2} \text {. }
$$

This implies Lemma 3.5.

g.e.d.

\section{Proof of Theorem B.}

We may assume $\sup |H|<\infty$. Let $\rho$ be the scalar curvature of $M$ and let $\beta$ be the second fundamental form of the immersion $f: M \rightarrow N$. Then by the Gauss equation we have

$$
\begin{aligned}
& m(m-1) b \geqq \rho-m^{2}|H|^{2}+|\beta|^{2}, \\
& \sup \left|K_{N}\right|+2 \sup |\beta|^{2} \geqq\left|K_{M}\right| .
\end{aligned}
$$

Since $\rho$ has a lower bound, the above inequalities imply the boundedness of the sectional curvature $K_{M}$.

Let $\phi: \tau(P, \lambda) \rightarrow P$ be the canonical projection and let $F: M \rightarrow \boldsymbol{R}$ be the smooth function defined by

$$
F(x)=\frac{1}{2}\{d(f(x), \phi f(x))\}^{2},
$$

where $d($,$) is the distance function on N$. Since $M$ is a complete Riemannian manifold whose sectional curvature is bounded, [5, Theorem $\left.\mathrm{A}^{\prime}\right]$ implies the existence of a sequence $\left\{x_{k}\right\}_{k=1}^{\infty}$ in $M$ such that

$$
|\operatorname{grad} F|\left(x_{k}\right)<1 / k \text {, }
$$




$$
\lim _{k \rightarrow \infty} F\left(x_{k}\right)=\sup F,
$$

where $\nabla^{2} F$ denotes the Hessian of $F$ with respect to the Riemannian metric of $M$. We set $\lambda_{k}=d\left(f\left(x_{k}\right), \psi f\left(x_{k}\right)\right)$. Then (4.3) implies $\lim _{k \rightarrow \infty} \lambda_{k}=\lambda$. Since $\lambda>0$, we may assume $0<\lambda_{k} \leqq \lambda$ for all $k$. Let $\sigma_{k}:\left[0, \lambda_{k}\right] \rightarrow N$ be the geodesic parametrized by arclength such that $\sigma_{k}(0)=\phi f\left(x_{k}\right)$ and $\sigma_{k}\left(\lambda_{k}\right)=f\left(x_{k}\right)$. Then $\dot{\sigma}_{k}(0)$ is perpendicular to $P$. Let $\left\{e_{1}, \cdots, e_{m}\right\}$ be an orthonormal basis of $T_{x_{k}} M$ such that $V^{1}\left(P, \sigma_{k}\right)$-component of $f_{*} e_{j}$ vanishes for all $j>p$. We set as follows:

$$
\begin{gathered}
h_{i}(k)=h_{i}\left(b, \mu, \lambda_{k}\right), \\
c_{j}^{2}(k)=\mid V^{\imath}\left(P, \sigma_{k}\right) \text {-component of }\left.f_{*} e_{j}\right|^{2} .
\end{gathered}
$$

LEMMA 4.1. $\sum_{i=0}^{2} \sum_{j=1}^{m} h_{i}(k) c_{j}^{\imath}(k) \geqq p h_{1}(k)+(m-p) h_{2}(k)+h(k)$ ， where $h(k)=-\left\{p\left|h_{0}(k)-h_{1}(k)\right|+(m-p)\left|h_{0}(k)-h_{2}(k)\right|\right\} /\left(k \lambda_{k}\right)^{2}$.

Proof. For convenience, put $h_{\imath}=h_{i}(k)$ and $c_{\jmath}^{\imath}=c_{\jmath}^{\imath}(k)$. Since $c_{\jmath}^{1}=0(j>p)$, $\sum_{\imath=0}^{2} c_{\jmath}^{\imath}=1$ and $h_{2} \geqq h_{1}$, we see that

$$
\begin{aligned}
\sum_{i=0}^{2} \sum_{j=1}^{m} h_{i} c_{j}^{2} & =\sum_{j=1}^{p}\left\{h_{0} c_{j}^{0}+h_{1}\left(1-c_{j}^{0}-c_{j}^{2}\right)+h_{2} c_{j}^{2}\right\}+\sum_{p<j}\left\{h_{0} c_{j}^{0}+h_{2}\left(1-c_{j}^{0}\right)\right\}, \\
& \geqq \sum_{j=1}^{p}\left\{h_{1}+\left(h_{0}-h_{1}\right) c_{j}^{0}\right\}+\sum_{p<j}\left\{h_{2}+\left(h_{0}-h_{2}\right) c_{j}^{0}\right\} .
\end{aligned}
$$

Since $\left\langle\operatorname{grad} F, e_{\jmath}\right\rangle=\lambda_{k}\left\langle\dot{\sigma}_{k}\left(\lambda_{k}\right), f_{*} e_{\jmath}\right\rangle$, (4.1) implies $c_{j}^{0}(k)<1 /\left(k \lambda_{k}\right)^{2}$. Hence we have

$$
\sum_{i=0}^{2} \sum_{j=1}^{m} h_{i} c_{j}^{2} \geqq p\left\{h_{1}-\left|h_{0}-h_{1}\right| /\left(k \lambda_{k}\right)^{2}\right\}+(m-p)\left\{h_{2}-\left|h_{0}-h_{2}\right| /\left(k \lambda_{k}\right)^{2}\right\} .
$$

q. e. d.

Let $I_{k}$ be the index form for the pair $\left(P, \sigma_{k}\right)$. Then a calculation shows that

$$
\frac{1}{\lambda_{k}} \nabla^{2} F\left(e_{\jmath}, e_{j}\right)=\left\langle\beta\left(e_{\jmath}, e_{j}\right), \sigma_{k}\left(\lambda_{k}\right)\right\rangle+I_{k}\left(J_{\jmath}, J_{j}\right),
$$

where $J_{\jmath}$ is the $P$-Jacobi field along $\sigma_{k}$ such that $J\left(\lambda_{k}\right)=f_{*} e_{j}$. Applying Lemma 3.5 to the pair $\left(P, \sigma_{k}\right)$, we have

$$
I_{k}\left(J_{\jmath}, J_{\jmath}\right) \geqq \sum_{\imath=0}^{2} h_{i}(k) c_{j}^{\imath}(k) .
$$

Hence (4.2), (4.4), (4.5) and Lemma 4.1 imply

$$
m / k \lambda_{k} \geqq-m \sup |H|+p h_{1}(k)+(m-p) h_{2}(k)+h(k) .
$$


Since $\lim _{k \rightarrow \infty} h_{\imath}(k)=h_{i}(b, \mu, \lambda)$ and $\lim _{k \rightarrow \infty} h(k)=0$, we have

$$
\sup |H| \geqq \frac{p}{m} h_{1}(b, \mu, \lambda)+\frac{m-p}{m} h_{2}(b, \mu, \lambda) .
$$

This completes the proof of Theorem B.

\section{REFERENCES}

[1] R. Bishop And R. Crittenden, Geometry of manifold, Academic Press, New York, 1964.

[2] L. Jorge AND D. Koutroufiotis, An estimate for the curvature of bounded sul). manifolds, Amer. J. Math., 103 (1981), 711-725.

[3] L. Jorge AND F. XAvier, A complete minimal surface in $R^{3}$ between two parallel planes, Ann. Math., 112 (1980), 203-206.

[4] L. JoRge AND F. XAVIER, An inequality between the exterior diameter and the mean curvature of bounded immersions, Math. Z., 178 (1981), 77-82.

[5] H. OMORI, Isometric immersions of Riemannian manifolds, J. Math. Soc. Japan, 19 (1967), 205-214.

Department of Mathematicis

UTSUNOMIYA UNIVERSITY

Mine-MACHi, UtSUNOMIYA, 321 JAPAN 